\title{
Méthodologie de transfert d'information pluviométrique en information hydrométrique. Application à la réestimation des risques d'étiage
}

\author{
A rainfall-to-hydrometry data-conversion methodology \\ and its use for closer estimation of low-water flow probability
}

\author{
Christian Michelet \\ Ingénieur civil \\ Ecole Nationale des Ponts et Chaussées
}

\section{Introduction}

L'aménagement des cours d'eau demande une connaissance aussi précise que possible du comportement des rivières, et en particulier du régime des basses eaux, appelé également "étiage". Il est fondamental de pouvoir calculer le risque d'apparition de ce phénomène, en répondant par exemple à la question suivante :

"Quelle est la probabilité pour que le débit reste sans interruption pendant une période de temps donnée, infé. rieur à un seuil en débit $Q_{0}$ fixé ?".

Lorsque l'on dispose de suffisamment d'informations sur les débits journaliers, les méthodes usuelles de modélisation des indicateurs d'étiage permettent de résoudre rapidement ce problème, en évaluant les risques d'étiage avec un intervalle de confiance associé à la prévision, suffisamment restreint.

Cependant, lorsque l'information hydrométrique est trop limitée, il devient indispensable de faire appel à un complément d'infornation. Aussi est-il naturel, dans le cas présent, de penser à l'information pluviométrique, tant la dépendance entre les épisodes de sécheresse et ceux d'étiage est forte.

La prise en compte de la pluviométrie dans le but de réestimer les risques d'étiage, a fait l'objet d'une étude de méthodologie de transfert d'information pluviométrique en information hydrométrique, permettant de reconstituer l'étiage à l'échelle journalière.

Le modèle mis au point a été testé, dans le cas d'un bassin versant de superficie limitée $\left(1170 \mathrm{~km}^{2}\right)$ sur la Sioule affluent de l'Allier, à partir des données suivantes:

- 18 années de mesures de débit moyen journalier à la station de Pont du Bouchet,

- 36 années de mesures de pluie totale journalière à la station de Montel de Gelat (dont 18 années concomitantes avec la série des mesures de débit).
Qu'est-ce que l'étiage?

L'étiage est par définition l'état de faible débit ; il est conditionné par deux facteurs, d'une part l'absence de précipitation, ou sécheresse, et d'autre part le tarissement des nappes souterraines.

Dans la relation de dépendance entre les pluies et le débit en matière d'étiage, on peut discerner deux aspects :

- la chronologie ou distribution temporelle des épisodes pluvieux,

- la persistance ou effet d'inertie des nappes.

Au niveau de la description statistique de l'étiage, on se limite aux indicateurs d'étiages "en terme de durée" (on dit aussi à débit fixé). C'est-à-dire que l'on fixe un seuil en débit $Q_{0}$, et on considère la segmentation d'une année obtenue, en faisant apparaître les périodes où le débit est resté inférieur au seuil $Q_{0}$ (cf. fig. 1).

On définit ainsi les indicateurs suivants :

- Durées individualisées de seuil $Q_{0}=$ série des durées des périodes de l'année pendant lesquelles le débit reste sans interruption inférieur à $Q_{0}$.

- Durée classée annuelle de seuil $Q_{0}$ : somme des durées individualisées ou nombre de jours pendant une année où le débit est inférieur à $Q_{0}$.

- Durée maximale annuelle de seuil $Q_{0}$ : durée de la plus longue période de l'année pendant laquelle le débit reste sans interruption inférieur à $Q_{0}$.

Principe du modèle de transfert d'information pluviomètrique en information hydrométrique

Pour décrire l'étiage à l'échelle journalière, en conservant la possibilité de reconstituer les indicateurs d'étiage en teme de durée, il suffit de savoir si le débit moyen journalier $Q(j)$ du jour $j$ se situe en-dessus ou en-dessous 


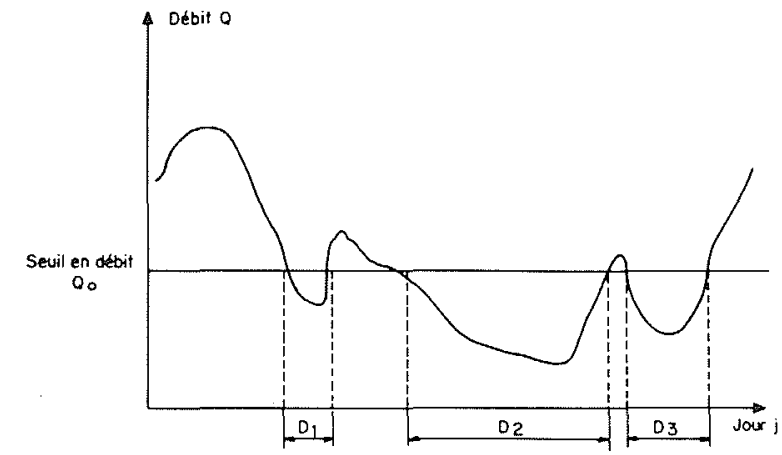

Figure 1 - durée classée $=D_{1}+D_{2}+D_{3} ;$ dutées individualisées $=\left(D_{1}, D_{2}, D_{3}\right)$; durée maximale $=\operatorname{Max}\left(D_{1}, D_{2}\right.$, $D_{3}$ ).

du seuil en débit $Q_{0}$ fixé. Cette information élémentaire que nous appellerons "état de seuil $Q_{0}$ ", est donc définie de la manière suivante:

- si $Q(j)>Q_{0}$ alors le jour $j$ est un état d'abondance de seuil $Q_{0}: E(j)=+1$

- si $Q(j) \leqslant Q_{0}$ alors le jour $j$ est un état de déficit de seuil $Q_{0}: E(j)=-1$.

En se limitant dans la description de l'étiage à l'échelle fine, à la donnée d'une variable discrète $E(j)$ à deux états, on peut ramener le problème de transfert d'information pluviométrique en information hydrométrique, à un problème décisionnel :

- choisir, sur la base de l'observation au jour $j$ du passé pluvieux, entre l'état de seuil $Q_{0}$ de déficit ou bien l'état de seuil $Q_{0}$ d'abondance.

Dans un premier temps, on modélise le passé pluvieux au jour $j$ par une famille d'indicateurs pluviométriques journaliers. Ensuite, on représente l'information disponible sur les séries concomitantes en pluie et en débit, pour un seuil $Q_{0}$ fixé, par un nuage de points dans l'espace $\mathrm{R}^{n}$ des paramètres pluviométriques, en convenant de figurer par un ' 0 ", respectivement par une " $X$ ", les jours correspondant à un état d'abondance, respectivement de déficit de seuil $Q_{0}$ (cf. figure 2). En faisant appel aux techniques de l'analyse des données multidimensionnelles, on va successivement :

- analyser de façon descriptive la répartition des états de seuil $Q_{0}$-abondance et débit- dans l'espace des paramètres pluviométriques, pour mettre en évidence une discrimination entre états de déficit et états d'abondance;

- décrire de manière probabiliste la distribution des états de seuil $Q_{0}$, et définir sur la base de cette description des règles de décision permettant de choisir l'état de seuil $Q_{0}$ du jour $j$ en fonction de la position du jour $j$ dans l'espace des paramètres du modèle.

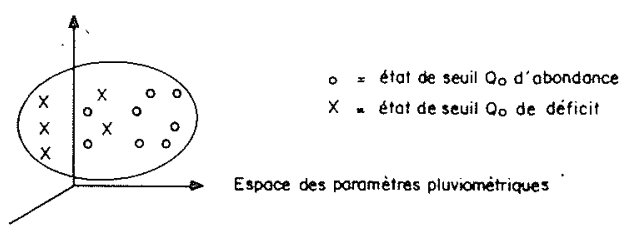

Figure 2 - Nuage "abondance-déficit" ou visualisation de la variable état de seuil $Q_{0}, E(j)$ dans l'espace des paramètres pluviométriques.
Analyse descriptive de la répartition des états de seuil $Q_{0}$ dans l'espace des paramètres pluviométriques

\section{Choix des paramètres pluviométriques}

Pour décrire l'étiage à partir de l'information de base constituée par la donnée des mesures de pluie totale journalière antérieure au jour $j$, on a défini 18 indicateurs pluviométriques, permettant de résumer le passé pluvieux d'une façon la plus exhaustive possible :

\section{- 9 Indicateurs "përiodiques"}

On se limite aux deux plus récentes périodes sèches, resp. pluvieuses, antérieures au jour $j$, et on considère pour chaque événement la durée de la période sèche, resp. la durée, la pluie maximale, la pluie moyenne de la période pluvieuse. On définit également un indicateur indiquant si le jour $j$ appartient à une période sèche ou pluvieuse.

\section{- 6 Indicateurs "de tendance"}

Sous forme de moyenne mobile des pluies journalières sur $1,3,10,30,60,90$ jours antérieurs au jour $j$, on prend en compte les tendances à court, moyen et long terme du passé pluvieux.

\section{- 2 Indicateurs "chronologiques"}

On caractérise la pluviosité respectivement la distri. bution des épisodes pluvieux, pendant 30 jours antérieurs au jour $j$, par le pourcentage de jours de pluie respectivement le nombre d'événements pluvieux pen. dant cette période.

\section{- 1 Indicateur de "précipitation neigeuse"}

On prend en compte l'influence particulière des chutes de neige, par la durée séparant une précipitation neigeuse de la précipitation non neigeuse qui lui succède.

\section{Analyse descriptive}

Si l'on considère le nuage "abondance-déficit", correspondant aux observations journalières de la période de concomitance, dans l'espace des paramètres pluviométriques, on veut pouvoir mettre en évidence une discrimination entre états d'abondance et de déficit, tout en se limitant à des projections particulières sur des sous-espaces de faible dimension.

\section{Critère de projection.}

On s'intéresse à deux types de visualisation du nuage de points abondance et déficit sur un axe ou sur un plan :

- celles qui donnent la meilleure vue d'ensemble du nuage,

- celles qui séparent le mieux les deux classes d'état abondance et déficit.

La première approche relève de l'analyse en composantes principales du nuage, car vouloir représenter le nuage dans son plus grand étalement, revient à chercher

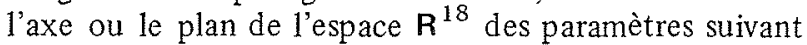
lesquels, en projection, l'inertie du nuage est maximale. 
La seconde approche fait appel à l'analyse factorielle discriminante : l'axe qui discrimine le mieux en projection les états d'abondance et de déficit est l'axe qui vérifie les deux conditions suivantes:

- les centres de gravité en projection sont le plus séparés possible,

- la dispersion respective de chaque classe autour de son centre de gravité est la plus faible possible.

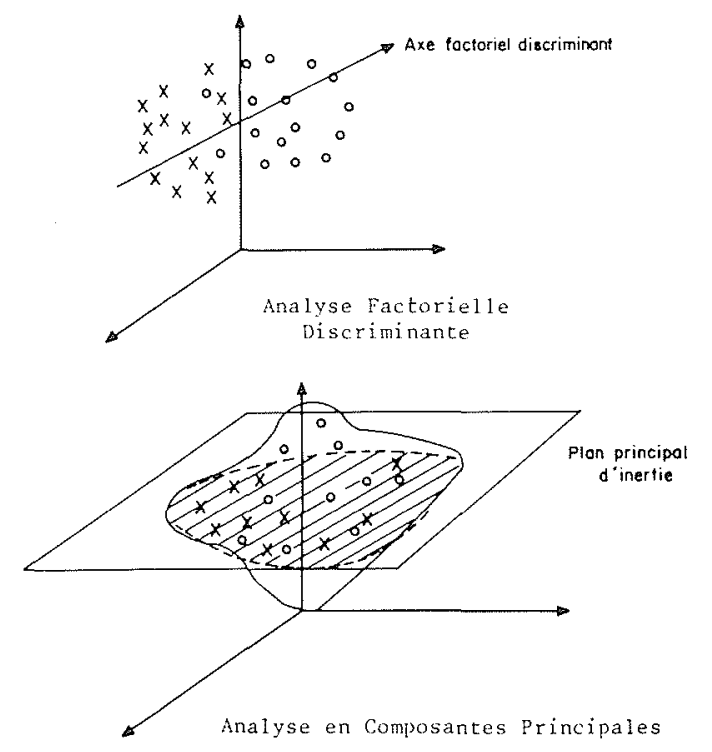

\section{Analyse comparative des deux approches}

Dans la mesure où on a défini seulement deux classes abondance-déficit, l'analyse factorielle discriminante met en évidence un unique axe factoriel discriminant (il n'existe pas de plan factoriel discriminant) ; cet axe vérifie a priori un certain optimum au niveau de la discrimination. Au contraire, l'analyse en composantes principales permet d'exhiber des plans factoriels dont le pouvoir de discrimination apparait a posteriori ; dans le cas présent le premier plan principal est également le plan qui permet la meilleure discrimination, ce qui traduit le fait que les facteurs principaux du passé pluvieux décrivent bien de façon implicite l'état de seuil.

Remarque : La recherche des plans principaux d'inertie est effectuée sur la série longue des pluies alors que la recherche de l'axe factoriel discriminant est effectuée sur la série concomitante seulement.

Exemples de résultats obtenus par analyse descriptive :

Figure 3 : Fluctuation respective du débit $Q(j)$ et de la projection du point représentatif du jour $j$ sur l'axe factoriel discriminant pour l'année 1961 et le seuil $Q_{0}=5 \mathrm{~m}^{3} / \mathrm{s}$,

Figure 4 : Projection de l'année 1961 sur le plan principal discriminant pour le seuil $Q_{0}=5 \mathrm{~m}^{3} / \mathrm{s}$.

Figure 5: Fluctuation respective du débit $Q(j)$ et de la projection du point représentatif du jour $j$ sur l'axe principal d'inertie le plus discriminant pour l'année 1961 et le seuil $Q_{0}=5 \mathrm{~m}^{3} / \mathrm{s}$.

\section{Conclusion de l'analyse descriptive}

L'analyse en composantes principales permet de mettre en évidence sur le premier plan factoriel princi- pal, une discrimination assez forte entre la classe des états de seuil $Q_{0}$ d'abondance et celle des états de seuil $Q_{0}$ de déficit ; cependant il n'apparait pas de structure spécifique pour la distribution des états journaliers de seuil $Q_{0}$ (de type gaussien par exemple).

L'analyse factorielle discriminante permet d'exhiber un axe factoriel discriminant, qui en dépit de son optimalité a priori, fournit un élément de décision moins riche que l'analyse en composantes principales dans la mesure où on se limite à une projection du nuage abondance-déficit sur un axe au lieu d'un plan. Ainsi, pour l'établissement du processus décisionnel de choix de l'état de seuil $Q_{0}$, à partir de la connaissance du passé pluvieux au jour $j$, on décide de résumer ce passé par la donnée de la position du point représentatif du jour $j$ en projection sur la plan des deux premiers facteurs pluviométriques principaux.

\section{Description probabiliste de la répartition des états de seuil $Q_{0}$ sur le plan factoriel discriminant}

Pour reconstituer un état de seuil $Q_{0}$ journalier à partir du passé pluvieux défini par les 18 paramètres, il ne suffit pas de décrire de façon qualitative, la répartition des états de déficit et d'abondance en observant la projection en plan du nuage abondance-déficit sur un plan factoriel discriminant, encore faut-il quantifier de façon probabiliste, le pouvoir discriminant du couple de facteurs pluviométriques obtenus par l'analyse descriptive du nuage des observations de la série concominante en pluie et en débit.

On définit la description probabiliste de la répartition des états de seuil $Q_{0}$, sur le plan des facteurs pluviométriques de la façon suivante :

- on considère une régionalisation du plan des facteurs à l'aide de deux faisceaux de droites verticales et horizontales (cf. figure 6),

- pour chaque région élémentaire ainsi constituée, on compte le nombre d'états d'abondance $N O$, de déficit $N X$ observés au cours de la période de concomitance, - on estime la "probabilité d'état de déficit" de chaque région, par la fréquence empirique des états de déficit $N X /(N X+N O)$.

En définitive, on obtient une carte de la distribution en probabilité de l'état de déficit sur le plan des facteurs, qui est d'autant plus précise que le nombre d'années de la période de concomitance est grand et le maillage est d'autant plus fin, de façon à ce que le nombre d'états observés dans chaque région soit représentatif. A titre d'exemple pour 18 années d'observations journalières, et un maillage $10 \times 10$, le nombre moyen d'états observés dans chaque région est égal à 70 .

On représente sur la figure 6 bis une carte de distribution en probabilité de l'état de déficit de seuil $Q_{0}$ avec les conventions de représentation suivantes:

\begin{tabular}{|c|c|c|c|c|c|}
\hline $\begin{array}{c}\text { probabilité } \\
\text { d'état de } \\
\text { déficit }\end{array}$ & $\begin{array}{c}\text { inférieur } \\
\text { à } 0,2\end{array}$ & $\begin{array}{c}\text { de } \\
0,2 \text { à } 0,4\end{array}$ & $\begin{array}{c}\text { de } \\
0,4 \text { à } 0,6\end{array}$ & $\begin{array}{c}\text { de } \\
0,6 \text { à } 0,8\end{array}$ & $\begin{array}{c}\text { plus de } \\
0,8\end{array}$ \\
\hline $\begin{array}{c}\text { représen- } \\
\text { tation }\end{array}$ & 8 & 0 & (blanc) & $x$ & $*$ \\
\hline
\end{tabular}

Remarque: le (blanc) représente également l'absence d'observations dans une région donnée. 


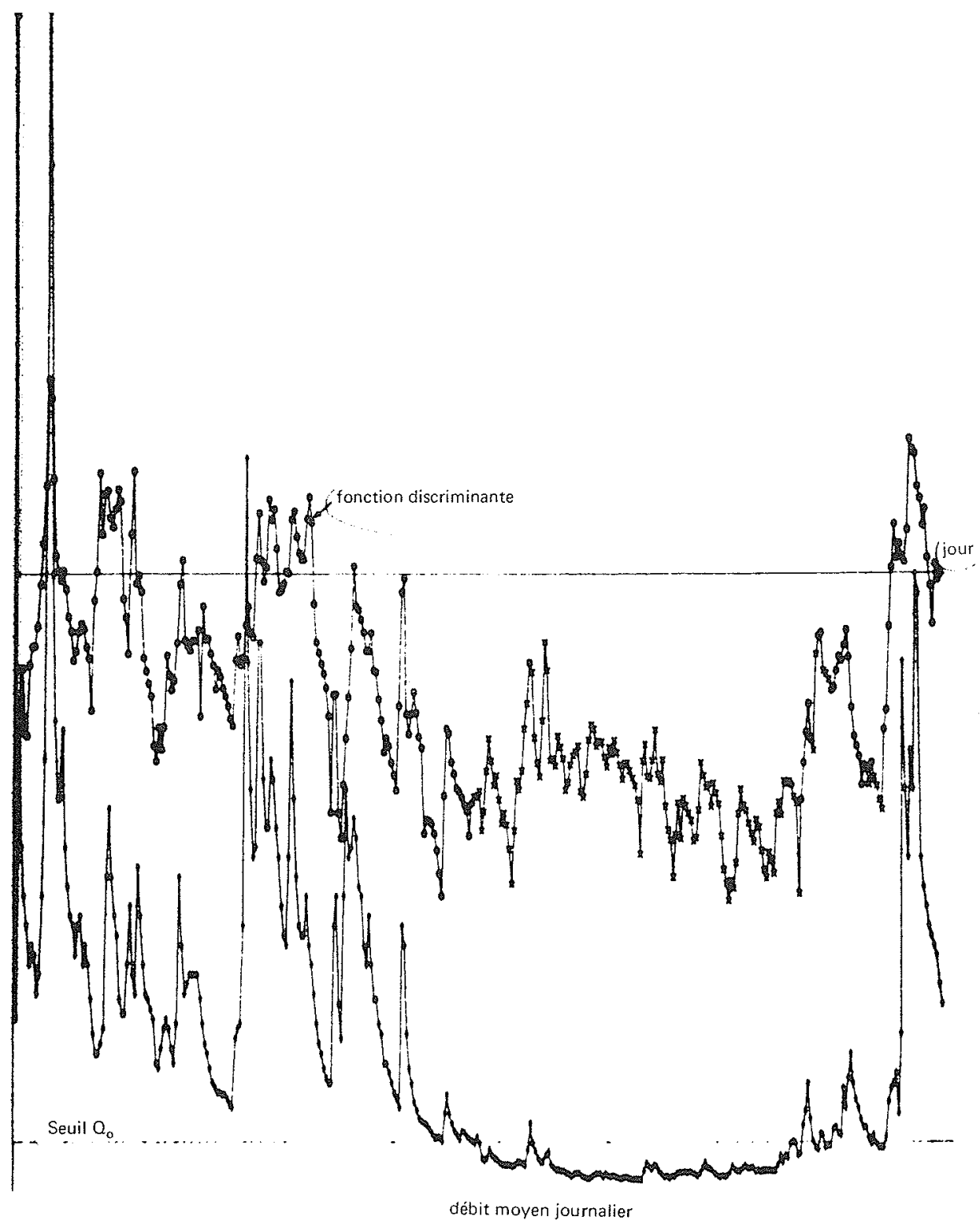

Figure 3 - Analyse factorielle discriminante. $Q_{0}=5 \mathrm{~m}^{3} \cdot \mathrm{s}^{-1}$. Année 61 (sèche).

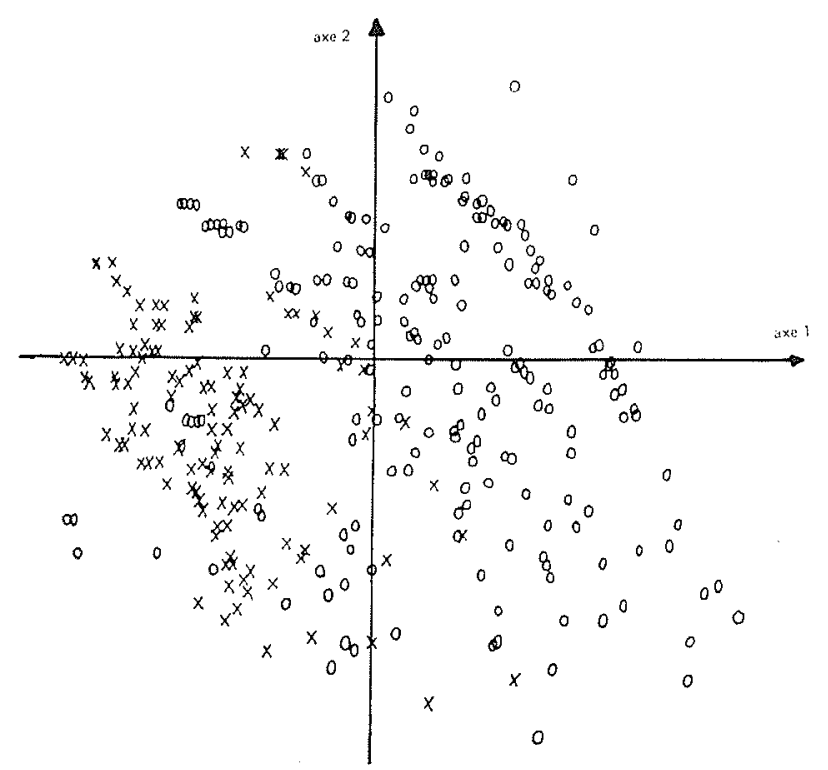

\section{Conclusion et remarques}

A partir de la description probabiliste de la répartition des états de seuil $Q_{0}$ de déficit sur le plan des deux facteurs pluviométriques discriminants observés au cours de la période de concomitance des informations en pluie et en débit, on est en mesure d'associer à chaque jour $j$, pour lequel on possède uniquement la donnée en pluie, à partir de sa position dans le plan des facteurs, la probabilité d'état de déficit du jour $j$. La chronologie des probabilités d'état de déficit jusqu'au jour $j$ va constituer l'élément décisionnel permettant de décider si le jour est en état d'abondance ou de déficit.

Dans le cas où le point représentatif du jour $j$ appartient à une région où aucune observation n'a été faite

Figure 4 - Plan discriminant 12 . Analyse en composantes principales. $Q_{0}=5 \mathrm{~m}^{3} \cdot \mathrm{s}^{-1}$. Année 61 (sèche). 


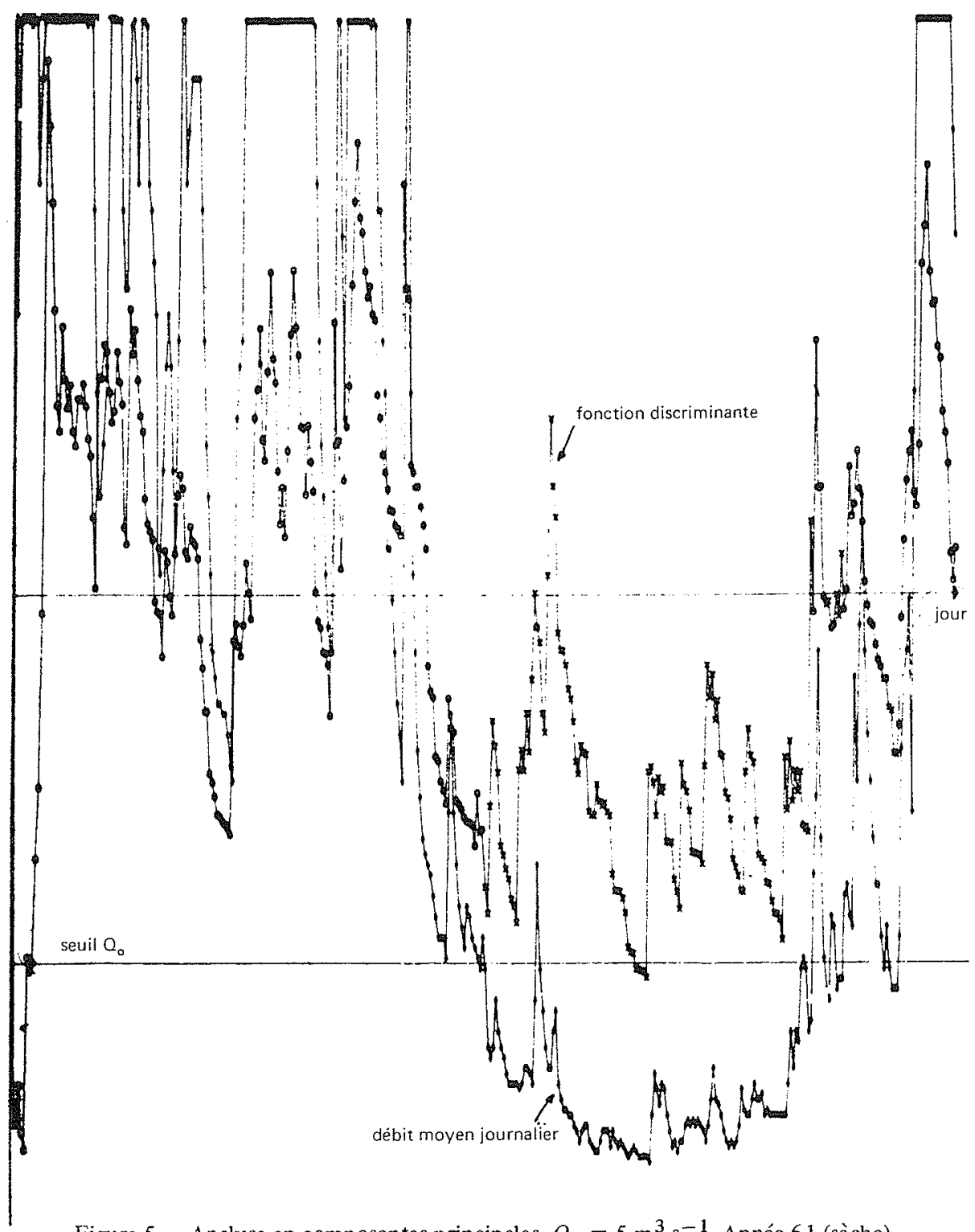

Figure 5 - Analyse en composantes principales. $Q_{0}=5 \mathrm{~m}^{3} \cdot \mathrm{s}^{-1}$. Année 61 (sèche).

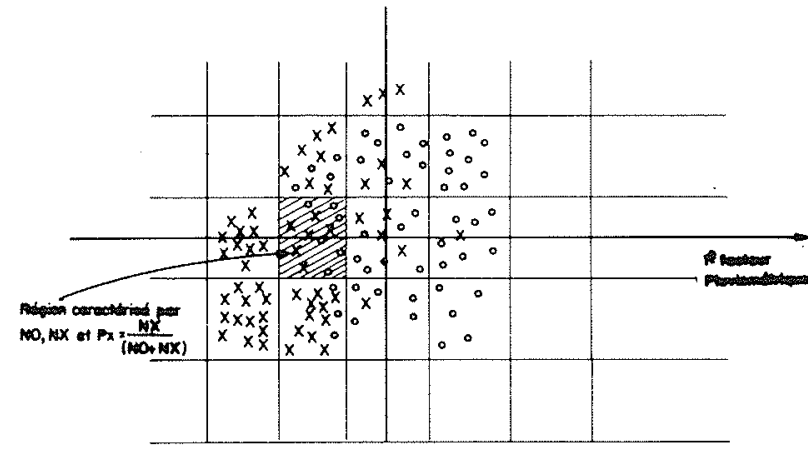

Figure 6 - Description probabiliste de la répartition des états de seuil $Q_{0}$ sur le plan factoriel discriminant.

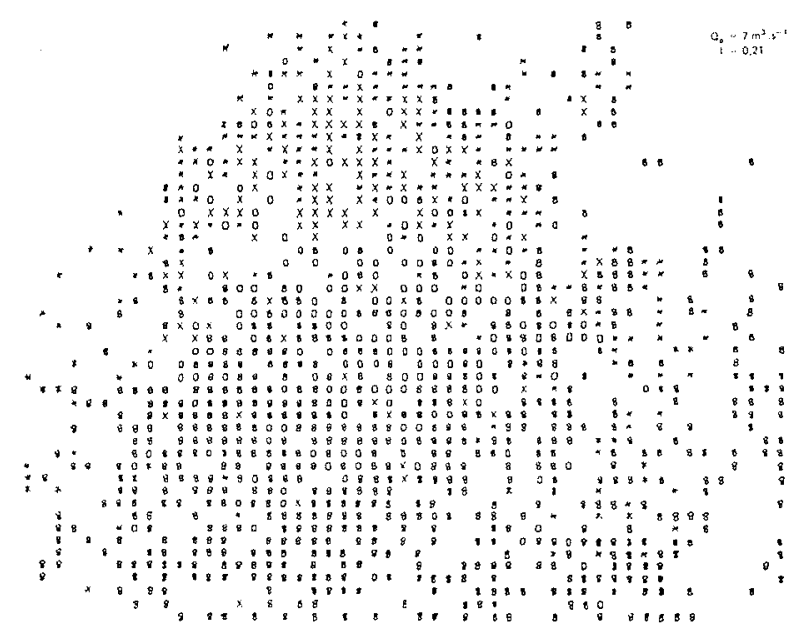

Figure 6 bis - Description probabiliste. Premier plan principal. 
au cours de la période de concomitance, un procédé d'extrapolation numérique permet d'estimer la probabilité d'état de déficit de la région considérée.

\section{Processus décisionnel de reconstitution de l'état de seuil $Q_{0}$ journalier}

Sur la base de la description probabiliste de la répartition des états de seuil $Q_{0}$, on définit des règles de décision ou stratégies permettant de choisir - à défaut de pouvoir l'identifier- l'état de seuil $Q_{0}$ en fonction de la position du jour $j$ et des jours précédents sur le plan des facteurs pluviométriques de l'analyse descriptive.

On appelle règle de décision permettant de déterminer l'état de seuil $Q_{0}$ du jour $j$ :

- toute application $\Gamma$ qui, à la donnée de la chronologie $C_{j}$ des probabilités d'état de déficit $p x(j)$, $p x(j-1), \ldots p x(j-n) \ldots$, associe un élément de l'espace des décisions $\Delta=\left\{\delta_{0}, \delta_{x}\right\}$, où $\delta_{0}$, respectivement $\delta_{x}$, représente la décision d'affecter au jour $j$ un état d'abondance respectivement de déficit de seuil $Q_{0}$.

$\Gamma: \mathrm{e} \longrightarrow \Delta$

$C_{j}=\left(p x(j), p x(j-1) \ldots(p x(j-n)) \rightarrow \delta_{0}\right.$ ou $\delta_{x}$

avec $\delta_{0}: j \rightarrow E(j)=1$ et $\delta_{x}: j \rightarrow E(j)=-1$

On a envisagé plusieurs types de stratégies élémentaires: stratégie déterministe $\Gamma_{1} /$

$$
\begin{aligned}
& \text { si } \left.p x(j) \in[0,0.5] \quad \Gamma_{1}\left(C_{j}\right)=\delta_{0}\right) \\
& \text { si } \left.p x(j) \in[0.5,1] \quad \Gamma_{1}\left(C_{j}\right)=\delta_{x}\right\}
\end{aligned}
$$

stratégie aléatoire $\Gamma_{2}$ /soit prob. la réalisation d'une variable aléatoire uniformément distribuée sur le segment $[0,1]$

$$
\begin{array}{ll}
\text { si } p x(j) \in[0, \text { prob. }] & \Gamma_{2}\left(C_{j}\right)=\delta_{0} \\
\text { si } p x(j) \in[\text { prob, } 1] & \Gamma_{2}\left(C_{j}\right)=\delta_{x}
\end{array}
$$

stratégie semi-déterministe $\Gamma_{3}$

$$
\begin{aligned}
& \text { Soit } \begin{array}{l}
p_{1} \text { et } p_{2} / 0<p_{1}<0,5<p_{2}<1 \\
\text { si } p x(j)<p_{1} \quad \Gamma\left(C_{j}\right)=\delta_{0}
\end{array} \\
& \text { Si } p_{1} \leqslant p x(j) \leqslant p_{2} \\
& \left\{\begin{array}{rr}
\text { si prob }>p x(j) & \Gamma\left(C_{j}\right)=\delta_{0} \\
\text { si } \operatorname{prob}<p x(j) & \Gamma\left(C_{j}\right)=\delta_{x} \\
\text { si } p x(j)>p_{2} & \Gamma\left(C_{j}\right)=\delta_{x}
\end{array}\right.
\end{aligned}
$$

Ces stratégies ont pris pour base de décision la valeur au jour $j$ de la probabilité d'état de déficit ; chaque jour est considéré comme indépendant des jours précédents ce qui, en matière d'étiage, est assez mal vérifié. La stratégie déterministe est trop rigide et au contraire la stratégie aléatoire est trop désordonnée; la stratégie semi-déterministe est un essai de conciliation entre les stratégies $\Gamma_{1}$ et $\Gamma_{2}$ à savoir que :

- d'une part, lorsque $p x(j)$ est au voisinage de 0 , respectivement de 1 , il est "intuitivement" presque certain que le jour $j$ sera un état d'abondance, respectivement de déficit ;
- d'autre part, lorsque $p x(j)$ est au voisinage de 0,5 , la décision est incertaine et l'application de la stratégie aléatoire sur le voisinage $\left[p_{1}, p_{2}\right]$ de 0,5 , est une façon de prendre en compte cette incertitude.

Ces stratégies élémentaires, par ce qu'elles considèrent isolément chaque état de seuil $Q_{0}$ à reconstituer, conduisent à une reconstitution assez médiocre des épisodes d'étiage, c'est-à-dire de la chronologie des états de seuil $Q_{0}$, aussi il apparaît nécessaire de définir des stratégies prenant en compte la dépendance entre l'affectation à un état de seuil $Q_{0}$ au jour $j$, et les décisions prises dans le passé.

\section{Stratégie semi-déterministe filtrée $\Gamma_{4}$}

On observe que la simulation de la chronologie des états de seuil $Q_{0}$, obtenue avec la stratégie semidéterministe $\Gamma_{3}$, engendre un grand nombre d'épisodes de courte durée en abondance ou en déficit, qui coupent des épisodes de plus longue durée et faussent ainsi la reconstitution des durées d'étiage. Le but de la stratégie $\Gamma_{4}$, est en l'occurrence de gommer ces changements d'états parasites.

Soit $\Gamma_{3}\left(C_{j}-4\right) \ldots \Gamma_{3}\left(C_{j}+2\right)$ les décisions pour les jours $j-4, j+2$.

Si l'on identifie $\delta_{x}$ à -1 et $\delta_{0}$ à 1 , on peut définir la somme

$$
S\left(C_{j}\right)=\sum_{k=j-4}^{k=j+2} \Gamma_{3}\left(C_{k}\right)
$$

$S\left(C_{j}\right)$ ne peut s'annuler car la sommation s'effectue sur un nombre impair de termes valant 1 ou -1 .

$$
\begin{array}{llll}
\text { Si } & S\left(C_{j}\right)>0 & \text { alors } & \Gamma_{4}\left(C_{j}\right)=\delta_{0} \\
\text { Si } & S\left(C_{j}\right)<0 & \text { alors } & \Gamma_{4}\left(C_{j}\right)=\delta_{x}
\end{array}
$$

\section{Stratégie déterministe de persistance $\Gamma_{5}$}

On prend en compte dans la décision d'affectation du jour $j$ à un état de seuil $Q_{0}$, la connaissance de l'état de seuil du jour précédent de la façon suivante :

$$
\text { Soit } \quad 0<p_{1}<0,5<p_{2}<1
$$

- si le jour $j-1$ est un état d'abondance de seuil $Q_{0}$

$$
\left\{\begin{array}{llll}
\text { si } & P x(j)>p_{2} & \text { alors } & \Gamma_{5}\left(C_{j}\right)=\delta_{x} \\
\text { si } & P x(j) \leqslant p_{2} & \text { alors } & \Gamma_{5}\left(C_{j}\right)=\delta_{0}
\end{array}\right.
$$

- si le jour $j-1$ est un état de déficit de seuil $Q_{0}$

$$
\left\{\begin{array}{llll}
\text { si } & P x(j)<p_{1} & \text { alors } & \Gamma_{5}\left(C_{j}\right)=\delta_{0} \\
\text { si } & P x(j) \geqslant p_{1} & \text { alors } & \Gamma_{5}\left(C_{j}\right)=\delta_{x}
\end{array}\right.
$$

\section{Conclusions et remarques}

Les stratégies $\Gamma_{4}$ et $\Gamma_{5}$ prennent en compte les décisions passées de façon différente, l'une par un procédé de moyenne mobile des décisions, la seconde en introduisant une forme de persistance, à l'aide des seuils en probabilité $p_{1}$ et $p_{2}$. 
Si l'on compare la chronologie des états de seuil $Q_{0}$ réels avec celle obtenue par simulation à l'aide des stratégies $\Gamma_{4}$ et $\Gamma_{5}$, on constate que si l'on peut se tromper sur la date du début des étiages, ou ne pas reconstituer les passages de très courtes durées au-dessus ou en dessous du seuil, on retrouve globalement assez bien les épisodes d'étiages.

Exemples de résultats obtenus par simulation: Figure 7: reconstitution de la chronologie des états de seuil $Q_{0}$ pour l'année 1964 et le seuil $Q_{0}=5 \mathrm{~m}^{3} / \mathrm{s}$ Figure 8: reconstitution de la chronologie des états de seuil $Q_{0}$ pour l'année 1964 et le seuil $Q_{0}=5 \mathrm{~m}^{3} / \mathrm{s}$.

Si l'on considère le critère d'erreur globale sur une année, dans la reconstitution de la chronologie des états de seuil $Q_{0}$, sur une année, on constate que la stratégie semi déterministe $\Gamma_{3}$ conduit à un taux de $20 \%$ d'erreurs, alors que la simulation avec les stratégies semi-déterministe filtrée et déterministe de persistance permettent de réduire à $10 \%$ le taux moyen d'erreur.

Il faut toutefois remarquer que dans la mesure où en fin de compte on désire reconstituer des statistiques d'indicateurs d'étiage, le risque d'erreur journalière importe moins que le risque d'erreur dans la reconstitution des durées des épisodes d'étiage.

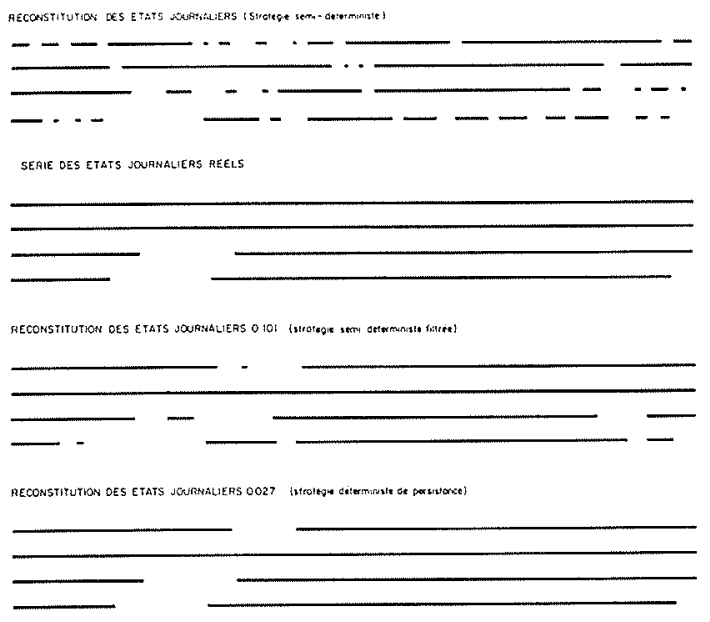

Figure 7 -

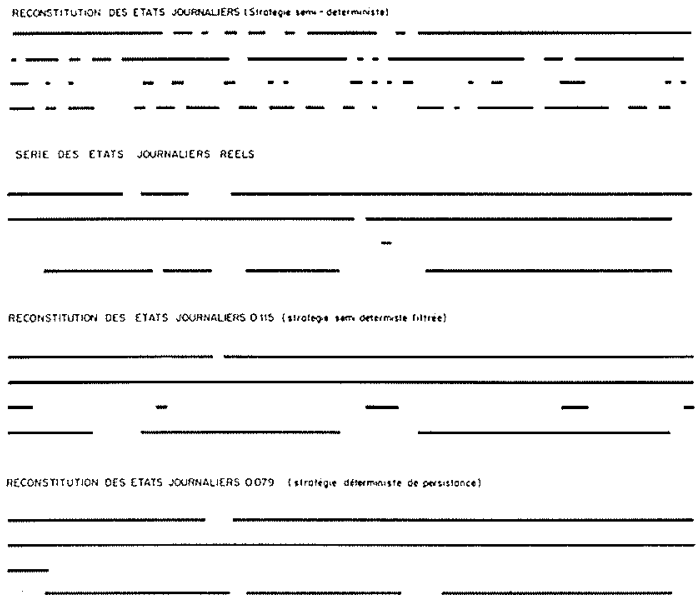

Figure 8
Reconstitution des indicateurs d'étiage et de leurs statistiques

A partir de la chronologie des états de seuil $Q_{0}$ reconstitués par simulation, on peut reconstituer les indicateurs d'étiage à débit $Q_{0}$ fixé :

- durée individualisée,

- durée classée annuelle,

- durée maximale annuelle.

On peut tester la valeur de la reconstitution en calculant le coefficient de corrélation $\rho$ entre séries d'indicateurs reconstitués et séries d'indicateurs réels, sur la période de concomitance. En prenant différents seuils en débit $Q_{0}$, on obtient une valeur moyenne $\rho_{m}$ :

$\rho_{m}=0,93$ pour la durée classée annuelle

$\rho_{m}=0,87$ pour la durée maximale annuelle

$\rho_{m}=0,78$ pour l'écart-type annuel des durées $\rho_{m}$ individualisées

$\rho_{m}=0,61$ pour le nombre annuel des durées individualisées.

Remarque: La moyenne annuelle des durées individualisées s'obtient à l'aide de la durée classée annuelle et du nombre d'étiages annuels.

On constate que les durées individualisées sont moins bien reconstituées que les durées maximales et classées, ce qui est imputable en partie à "I'effet de dentelle" naturel ou artificiel (simulation) qui consiste à couper des grands épisodes d'étiage ou d'abondance par un franchissement du seuil de très courte durée.

\section{Réestimation des risques d'étiage}

On prolonge la série des indicateurs réels, par la série des indicateurs reconstitués par simulation et réestimés par régression linéaire; on réévalue ainsi les paramètres des lois de probabilité des indicateurs d'étiage :

- nombre total d'étiages sur la période considérée, - moyenne et écart-type des durées individualisées, des durées classées et des durées maximales.

La réestimation des risques d'étiage des indicateurs annuels consiste à réestimer la valeur des durées $D^{m} Q_{0}$ correspondant à une période de retour de $m$ années, pour le seuil $Q_{0}$ donnée par la formule :

$$
D^{m} Q_{0} / \text { Prob. }\left(D Q_{0}>D^{m} Q_{0}\right)=\frac{1}{m}
$$

Pour les durées individualisées, on utilise la formule approchée suivante :

$$
D^{m} Q_{0} / \text { Prob. }\left(D Q_{0}>D^{m} Q_{0}\right)=\frac{1}{m} \times \frac{n}{N\left(Q_{0}\right)}
$$

$$
\begin{cases}n & \text { nombre d'années de la période considérée } \\ N\left(Q_{0}\right) & \text { nombre d'étiages de seuil } Q_{0} \text { durant la période } \\ & \text { considérée }\end{cases}
$$

Le résultat final de la réestimation des risques d'étiage s'observe en comparant les courbes débit-durée-fréquence $\left(Q_{0}, D^{m} Q_{0}\right)$ pour plusieurs valeurs de la période 
de retour $m(10,20,50$ èt 100 ans) obtenues à l'aide de la série courte en débit, avec celles obtenues sur la série longue en prenant en compte l'information pluviométrique. On peut apprécier de façon qualitative le gain d'information en comparant les intervalles de confiance associés à l'estimation des courbes đébit-durée-fréquence.

Exemples de résultats obtenus pour l'indicateur d'étiage "durée individualisée" :

Figure 9 : Courbe Intensité Durée Fréquence

Figure 9 bis: Intervalle de confiance des courbes Intensité Durée Fréquence

( pour la série courte de 18 années de mesures de débits.

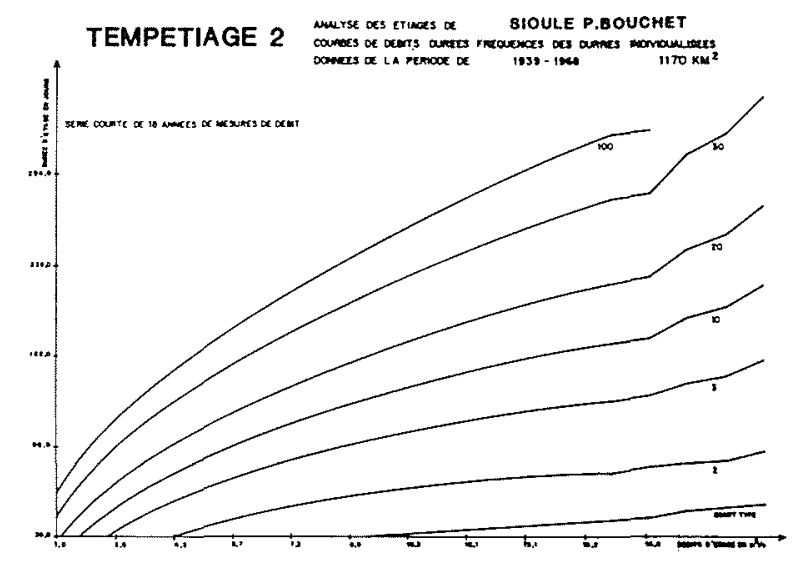

Figure 9 -

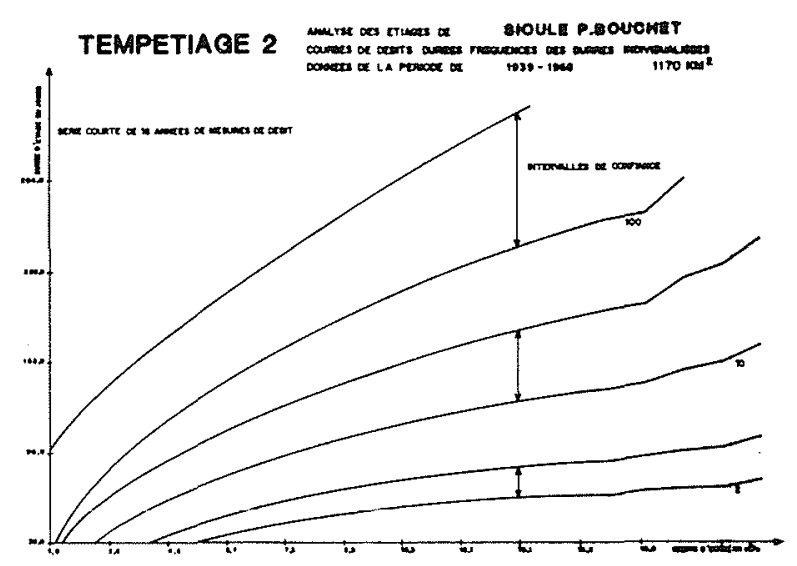

Figure 9 bis -

Figure 10: Courbe Intensité Durée Fréquence

Figure 10 bis : Intervalle de confiance des courbes Intensité Durée Fréquence

\{ pour la série prolongée de 34 années prenant en compte l'information pluviométrique.

\section{Conclusion}

En définitive, le modèle de transfert de l'information pluviométrique en information hydrométrique à l'échelle fine, se caractérise par trois points fondamentaux :
Principe

- On reconstitue une information journalière sur le débit, la plus élémentaire permettant de décrire l'étiage : l'état de seuil $Q_{0}$.

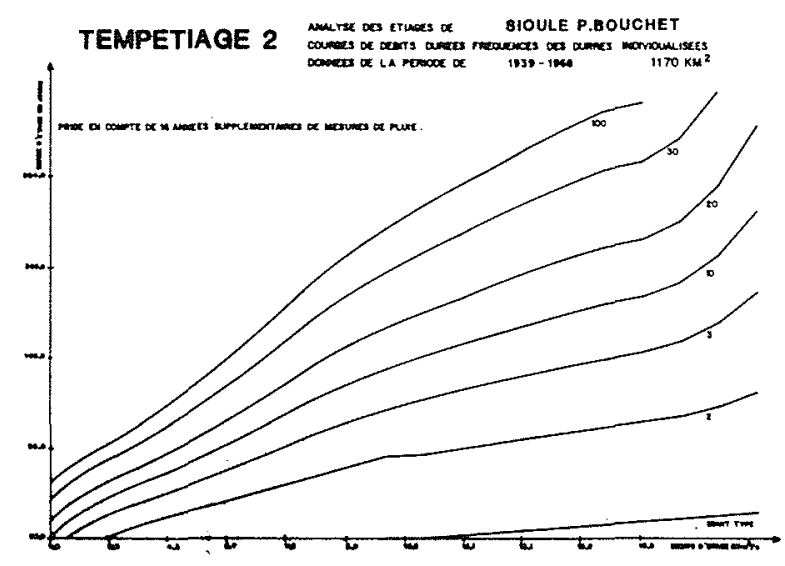

Figure 10 -

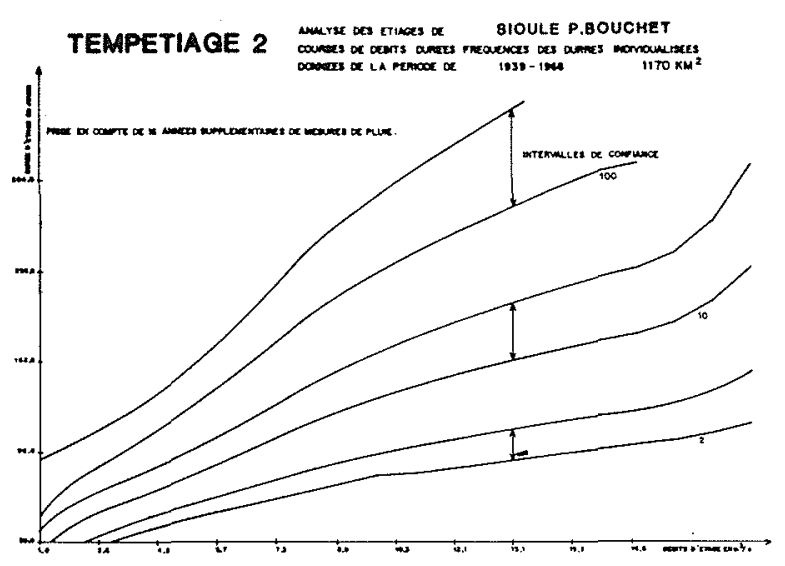

Figure 10 bis -

\section{Aspect descriptif}

- On étudie ensuite, par une analyse descriptive, comment les paramètres pluviométriques conditionnent l'état de seuil, dans quelle mesure ils induisent une régionalisation de l'espace des paramètres, associée à chacune des deux modalités - déficit, abondance de la variable état de seuil.

On met en évidence des facteurs pluviométriques optimum du point de vue de la discrimination de l'état d'abondance et de l'état de déficit.

\section{Aspect décisionnel}

- Après s'être restreint au plan formé par deux facteurs pluviométriques discriminants, on décrit la distribution en probabilité sur ce plan de la variable état de seuil.

A partir de la connaissance de la trajectoire décrite sur le plan des facteurs pluviométriques, et de la description probabiliste de ce plan, on reconstitue par simulation le processus des états de seuil $Q_{0}$ journaliers. Enfin on réestime les indicateurs d'étiage à débit $Q_{0}$ fixé et leurs statistiques. 
Si l'on compare les résultats obtenus pour ce qui est de la reconstitution des indicateurs d'étiage annuels, avec ceux que l'on aurait obtenus par régression linéaire multiple, on peut constater qu'ils sont au moins aussi bons et qu'on peut reconstituer en outre les durées individualisées.

On peut dire que, dans une certaine mesure, les paramètres pluviométriques choisis traduisent bien implicitement l'état de seuil $Q_{0}$ et que la description probabiliste ainsi que les règles de décisions définies, exploitent au maximum la discrimination des états d'abondance et de déficit dans l'espace des paramètres pluviométriques.
Le modèle fait l'objet d'études complémentaires visant à apporter des perfectionnements :

- au niveau de l'étude descriptive, par la recherche de plans factoriels discriminants obtenus par analyse factorielle discriminante du nuage des observations en distinguant plusieurs classes;

- au niveau de l'étude décisionnelle, par la recherche de stratégies plus élaborées.

Le modèle a été mis au point en quatre mois au L.N.H., dans le cadre d'un travail de fin d'étude à l'Ecole nationale des Ponts et Chaussées. 